Ann. Biol. anim. Bioch. Biophys., 1977, 17 (6), 997-1004.

\title{
Teneur en ADN de l'ovocyte de souris au stade préleptotène de condensation chromosomique
}

par Michèle HARTUNG, Catherine SOUCHIER *, A. STAHL

\author{
Laboratoire d'Hisfologie ef Embryologie II, \\ Faculté de Médecine, \\ 27, boulevard Jean-Moulin \\ 13385 Marseille Cedex 4. \\ * Laboratoire d'Histologie, Embryologie et Cytogénétique, \\ Faculté de Médecine, 8, avenue Rockefeller \\ 69373 Lyon Cedex 2.
}

Summary. DNA content of mouse oocyfe at the preleptotene stage of chromosome condensation.

Studies of meiotic onset in the mouse oocyte revealed the existence of a preleptotene stage of chromosome condensation. Densitometric studies of the condensation stage nucleus, stained by Feulgen reaction, showed that this nucleus contained 4C DNA. This observation compared with chronological analysis suggested that the condensation stage was situated between premeiotic S phase and leptotene.

L'existence d'un stade de condensation chromosomique préleptotène a été décrite chez des Insectes (pour revue voir Wilson, 1928) et chez de nombreux végétaux (pour revue voir Walters, 1972, 1976). II était passé inaperçu ou considéré comme dégénératif chez les Mammifères, jusqu'à sa description dans l'ovogenèse humaine (Stahl ef Luciani, 1971), dans l'ovogenèse de la lapine (Devictor-Vuillet et al., 1973) et dans celle de la brebis (Mauleon et al., 1976). L'étude cytologique des stades précoces de la méiose de l'ovocyłe de Souris a montré l'existence d'un stade de condensation dont les caractéristiques morphologiques sont particulièrement favorables à une analyse précise. Il est essentiel de situer dans le temps le stade de condensation par rapport au stade leptotène, et par rapport à la phase de synthèse de l'ADN qui précède les phénomènes morphologiques de la méiose.

\section{Matériel et méthodes.}

Les préparations cytologiques sont obtenues à partir de gonades d'embryons de Souris femelles âgées de 13 à 20 jours, prélevées et traitées selon la technique de Luciani ef al. (1974). Cette technique a l'avantage de fournir sur la même lame des 
noyaux de cellules germinales parfaitement étalés et des noyaux de cellules somatiques. Après fixation par le méthanol-acide acétique (3/1) les gonades sont placées dans de l'acide acétique à 45 p. 100 . Un pipettage rapide permet la dissociation cellulaire. Les cellules en suspension sont étalées sur lames préalablement glacées. Les lames sont colorées par la réaction de Feulgen : après hydrolyse dans $\mathrm{HCI} 5 \mathrm{~N}$ à $26^{\circ}$ pendant $15 \mathrm{mn}$, elles sont colorées par la pararosaniline pendant $1 \mathrm{~h}$ puis lavées dans trois bains d'eau sulfureuse.

Les mesures sont effectuées à l'aide d'un analyseur d'images (Quantimet 720, Cambridge Instruments) équipé d'une caméra Plumbicon et d'un module de densitométrie (Fischer et Bond, 1972). Les noyaux sont sélectionnés et analysés en lumière monochromatique de 570 nanomètres de longueur d'onde, à l'aide d'un microscope Zeiss (Obj. à immersion $\times 100$ ) et filmés par la caméra Plumbicon. Le module densitométrique permet la mesure de la densité optique totale intégrée, correspondant à chaque noyau. Les mesures ont été faites sur la même préparation afin d'éviter les distorsions imputables aux facteurs techniques (intensité de coloration par la réaction de Feulgen, étalement des préparations).

Pour les cellules germinales, les mesures ont été effectuées au stade de condensation maximum. Les noyaux somatiques servant de référence $(2 \mathrm{C})$ ont été sélectionnés de façon à présenter la même taille et le même aspect chromatinien. Pour chaque noyau, les mesures ont été répétées 5 fois.

\section{Résultats.}

\section{A. Les aspects morphologiques.}

L'analyse des aspects observés permet de distinguer : 10 une phase de contraction progressive $; 2^{\circ}$ un stade de condensation maximum ; $3^{\circ}$ une phase de décondensation qui aboutit progressivement à la formation du noyau leptotène caractéristique.

Les processus de condensation débutent par l'apparition de filaments très fins et irréguliers, qui se ramassent peu à peu autour de chromocentres, constitués par l'hétérochromatine centromérique (fig. 1). Au maximum du stade de condensation, le noyau renferme 40 blocs chromosomiques d'aspect compact (fig. 2). La décondensation se traduit par la réapparition de filaments plus épais et plus réguliers qu'au début du stade de condensation (fig. 3). Ils s'allongent ensuite, en prenant progressivement l'aspect des chromosomes au stade leptotène (fig. 4).

Une numération des stades observés sur les préparations, portant chaque fois sur 200 cellules, montre déjà de nombreuses cellules germinales au stade de condensation, chez l'embryon de Souris de 13 jours. Leur nombre s'accroît à 14 jours. A 15 jours, les ovocytes qui se trouvent au stade de condensation sont très nombreux, mélangés à des ovocytes au stade leptotène. A 16 jours, on voit encore de nombreux ovocytes au stade de condensation mais les stades leptotène et zygotène sont prédominants. Le stade de condensation devient rare chez l'embryon de 17 jours et ne se voit plus à 18 jours, où les stades zygotène et pachytène sont prédominants (fig. 5). 

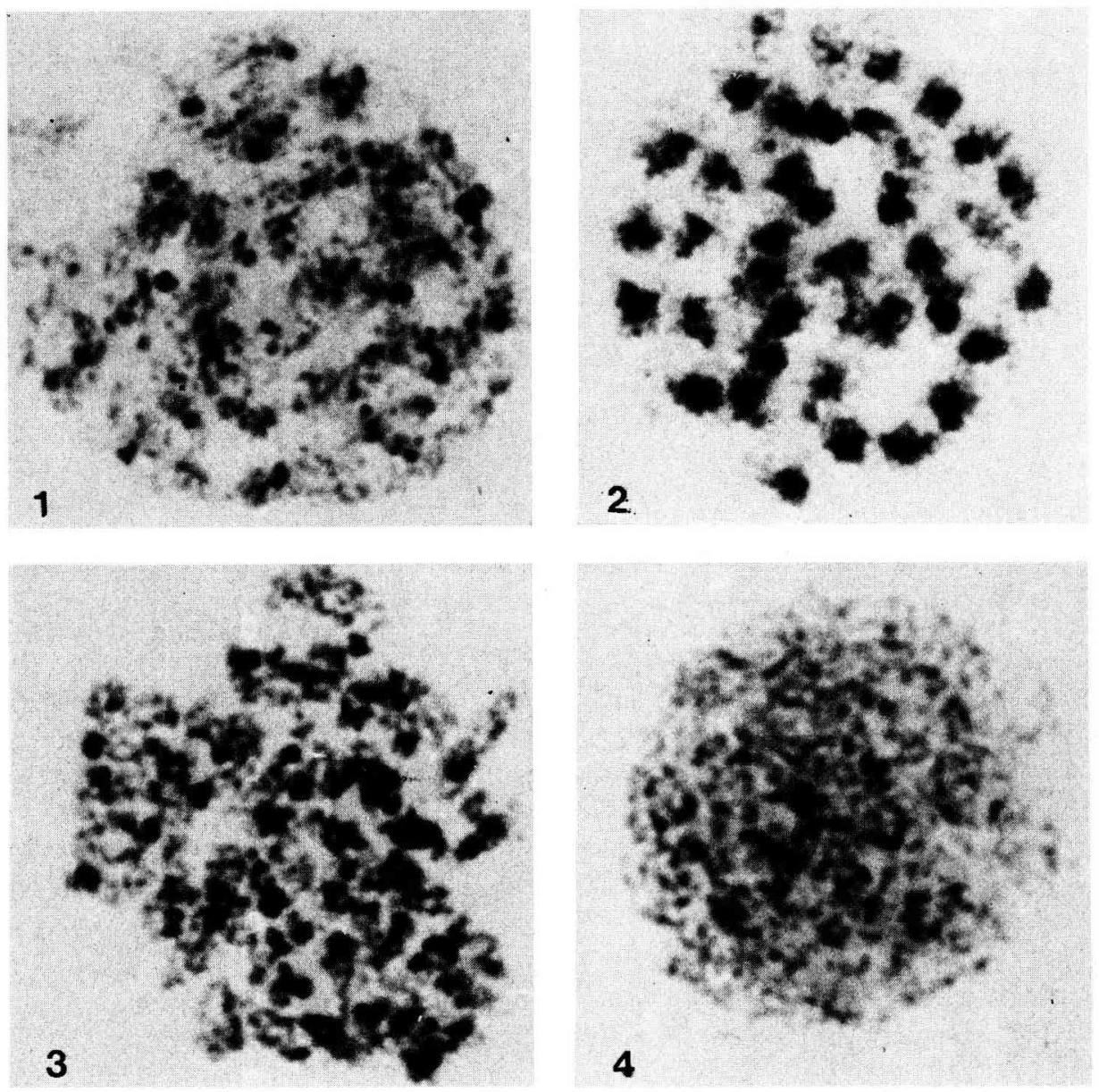

\section{PLANCHE I}

FIG. 1. - Noyau d'ovocyte de souris au début de la phase de condensation préleptotène : les filaments fins et irréguliers se ramassent autour des chromocentres. $(\times, 1800)$.

FIG. 2. - Ovocyte au stade de condensation maximum : le noyau renferme 40 blocs chromosomiques d'aspect compact. ( $(\times 1400)$.

FIG. 3. - Phase de décondensation : les filaments chromosomiques se reconstituent aux dépens de chaque masse. $(\times 2200)$.

FIG. 4. - Noyau d'ovocyfe de souris au stade leptotène. ( $(\times 2400)$. 


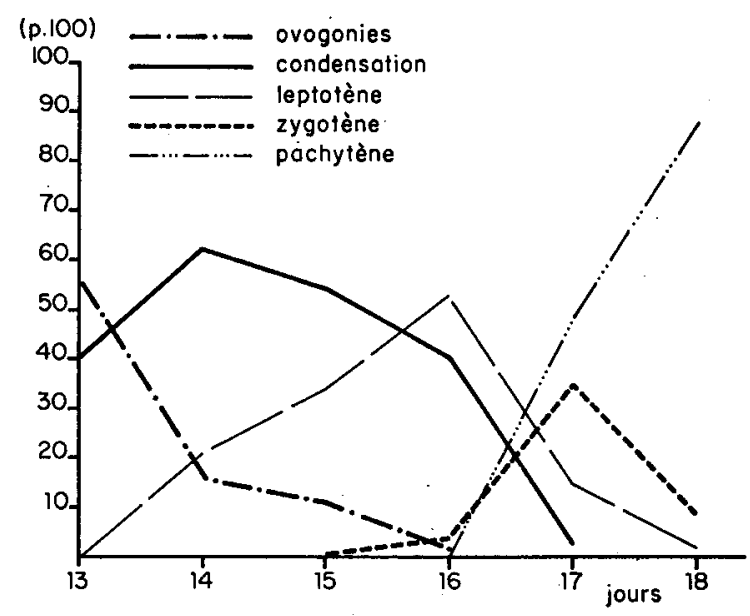

FIG. 5. - Proportions comparées des ovocyles au stade de condensation et des stades leptofène. zygolène ef pachytène chez l'embryon de Souris, du $13^{\mathrm{e}}$ au $18^{\mathrm{e}}$ jour.

\section{B. Efude densitométrique.}

Les valeurs moyennes de la densité optique intégrée de 40 cellules somatiques et de 40 cellules germinales au stade de consensation maximum sont présentées dans le tableau 1. L'unité de mesure retenue est le picogramme $\left(10^{-12} \mathrm{~g}\right)$. La quantité d'ADN des cellules somatiques de Souris est de 4,98 pg (McCarthy, 1969).

Les résultats de nos mesures sont exprimés en valeur densitométrique donnée par le Quantimet et en quantité d'ADN (en pg) calculée après la transformation suivante : $(X \times 4,98) / 4801$ où :

$X=$ valeur densitométrique donnée par le Quantimet.

4801 = valeur densitométrique moyenne des cellules somatiques (tabl. 1).

4,98 = quantité moyenne d'ADN (en pg) des cellules somatiques.

La valeur moyenne de la densité optique intégrée des cellules germinales au stade de condensation a été trouvée égale à 8980 , ce qui correspond à une valeur. moyenne de quantité d'ADN égale à 9,32 pg (tabl. 1).

La figure 6 donne une représentation graphique de ces résultats sous la forme de deux histogrammes. On note l'existence de deux modes : 5 pg pour les cellules somatiques, 10 pg pour les cellules germinales. La teneur en ADN des cellules somatiques étạnt de $4,98 \pm 1,18 \mathrm{pg}$, la teneur en $A D N$ des cellules germinales devrait être de $4,98 \times 2 \pm 1,18 \times 2=9,96 \pm 2,36$. Cing noyaux de cellules germinales se situent hors de cet intervalle. Ceci peut s'expliquer soit par la perte accidentelle de matériel chromosomique lors de l'étalement des cellules germinales, soit par le fait que certaines portions de filaments chromosomiques encore peu condensés ne sont pas détectées correctement lors des mesures. Au contraire les cellules somatiques possèdent une membrane nucléaire bien définie et un aspect plus homogène, ce qui facilite leur sélection. En tenant compte de ces difficultés, le chiffre réellement trouvé pour les cellules germinales $(9,32 \mathrm{pg})$ indique de façon satisfaisante que les ovocytes au stade de condensation contiennent $4 C$ ADN. 
TABLEAU 1

Densifé optique intégrée (unités arbitraires) et feneur en ADN

des cellules somatiques et des ovocytes au stade de condensation

\begin{tabular}{|c|c|c|c|}
\hline \multicolumn{2}{|c|}{ Cellules somatiques } & \multicolumn{2}{|c|}{$\begin{array}{c}\text { Ovocytes au stade de condensation } \\
\text { préleptolène }\end{array}$} \\
\hline $\begin{array}{c}\text { Densité } \\
\text { (Unités arbitraires) }\end{array}$ & $\begin{array}{l}\text { Quantité d'ADN } \\
\text { (pg) }\end{array}$ & $\begin{array}{c}\text { Densité } \\
\text { (Unités arbitraires) }\end{array}$ & $\begin{array}{l}\text { Quantité d'ADN } \\
\text { (pg) }\end{array}$ \\
\hline $\begin{array}{l}5100 \\
5100 \\
4700 \\
5380 \\
5486 \\
4150 \\
5050 \\
5260 \\
5400 \\
6100\end{array}$ & $\begin{array}{l}5,29 \\
5,29 \\
4,87 \\
5,58 \\
5,69 \\
4,30 \\
5,24 \\
5,46 \\
5,60 \\
6,33\end{array}$ & $\begin{array}{r}6770 \\
8687 \\
9790 \\
8811 \\
10426 \\
4924 \\
9940 \\
8998 \\
9630 \\
10530\end{array}$ & $\begin{array}{r}7,02 \\
9,01 \\
10,15 \\
9,14 \\
10,81 \\
5,11 \\
10,31 \\
9,33 \\
9,99 \\
10,92\end{array}$ \\
\hline $\begin{array}{l}5250 \\
5800 \\
4720 \\
5100 \\
4630 \\
4050 \\
5130 \\
4530 \\
5140 \\
4834\end{array}$ & $\begin{array}{l}5,45 \\
6,02 \\
4,89 \\
5,29 \\
4,80 \\
4,20 \\
5,32 \\
4,70 \\
5,33 \\
5,01\end{array}$ & $\begin{array}{r}10648 \\
8500 \\
9251 \\
8590 \\
8300 \\
10730 \\
10510 \\
6500 \\
9260 \\
9280\end{array}$ & $\begin{array}{r}11,04 \\
8,82 \\
9,60 \\
8,91 \\
8,61 \\
11,13 \\
10,90 \\
6,74 \\
9,71 \\
9,63\end{array}$ \\
\hline $\begin{array}{l}4490 \\
4090 \\
3800 \\
4780 \\
4526 \\
3980 \\
3800 \\
4650 \\
4540 \\
4240\end{array}$ & $\begin{array}{l}4,66 \\
4,21 \\
3,94 \\
4,96 \\
4,69 \\
4,13 \\
3,94 \\
4,82 \\
4,71 \\
4,40\end{array}$ & $\begin{array}{r}9600 \\
5800 \\
10090 \\
9143 \\
5700 \\
8976 \\
9400 \\
9780 \\
8701 \\
9415\end{array}$ & $\begin{array}{r}6,96 \\
6,02 \\
10,46 \\
9,48 \\
5,91 \\
9,31 \\
9,75 \\
10,14 \\
9,02 \\
9,76\end{array}$ \\
\hline $\begin{array}{l}4629 \\
4878 \\
4171 \\
4962 \\
5215 \\
6215 \\
4500 \\
3993 \\
4602 \\
5100\end{array}$ & $\begin{array}{l}4,80 \\
5,06 \\
4,33 \\
5,15 \\
5,41 \\
6,45 \\
4,67 \\
4,14 \\
4,77 \\
5,29\end{array}$ & $\begin{array}{r}9930 \\
8795 \\
9878 \\
7960 \\
8915 \\
10618 \\
9483 \\
9137 \\
8641 \\
9216\end{array}$ & $\begin{array}{r}10,30 \\
9,12 \\
10,24 \\
8,26 \\
9,25 \\
11,01 \\
9,84 \\
9,48 \\
8,96 \\
9,56\end{array}$ \\
\hline $\begin{array}{l}\bar{x}=4801 \\
\sigma=582\end{array}$ & $\begin{array}{l}4,98 \\
0,60\end{array}$ & $\begin{array}{l}\bar{x}=8980 \\
\sigma=1365\end{array}$ & $\begin{array}{l}9,32 \\
1,42\end{array}$ \\
\hline
\end{tabular}




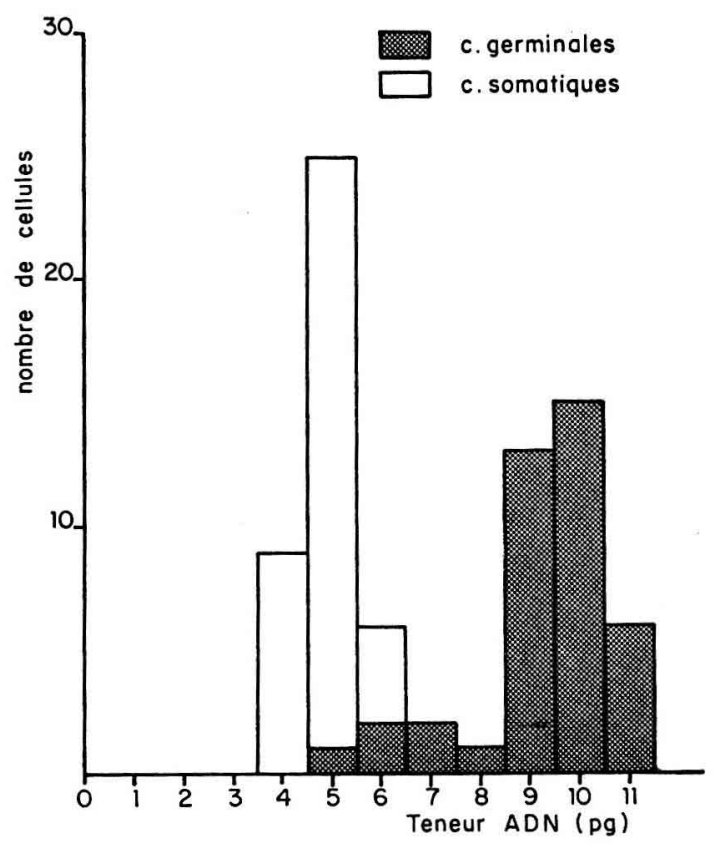

FIG. 6. - Distribution de la teneur en $A D N$ des cellules somafiques et des ovocyles au stade de condensation.

\section{Discussion.}

La fréquence comparée du stade de condensation entre le $13^{\mathrm{e}}$ ef le $18^{\mathrm{e}}$ jour, par rapport à celle des stades leptotène, zygotène et pachytène, suggère qu'il se déroule avant le début du stade leptotène. Cette conclusion est en accord avec les constatations de Mauleon ef al. (1976) chez la Brebis, basées sur l'incorporation de thymidine tritiée. Elle rejoint également celles des recherches chronologiques faites chez Lillium (Walters 1970, 1972 ; Bennett et Stern, 1975).

La teneur de 4C ADN des noyaux au stade de condensation exclut qu'il puisse s'agir de noyaux télophasiques ou de noyaux en phase G1. De même, si le stade de condensation correspondait à la phase $S$, on trouverait des teneurs en ADN intermédiaires entre $2 C$ et $4 C$. La morphologie de ces noyaux excluant la phase $G 2$, on est conduit à admettre qu'ils sont engagés dans une prophase. L'éventualité d'une prophase mitotique qui, selon Hilscher ef al. (1974) serait celle du dernier cycle mitotique des ovogonies, nous paraît devoir être écartée pour les raisons suivantes :

- L'étude ultrastructurale de ce stade révèle l'absence dans le cyłoplasme de l'ovocyte de centrioles et de microlubules (Hartung et Stahl, 1977);

- S'il s'agissait de la prophase de la dernière division mitotique des ovogonies, on devrait trouver au voisinage des groupes d'ovocytes au stade de condensation, des groupes de cellules en métaphase et en anaphase, ce qui n'est pas le cas (Mauleon ef al., 1976). 
L'ensemble de ces faits indique que le stade de condensation se situe entre la phase $S$ qui précède et conditionne les processus morphologiques de la méiose, et le stade leptotène.

La signification fonctionnelle du stade de condensation préleptotène n'est pas connue. Sa présence n'est pas constante chez toutes les espèces. Chez celles où ce stade existe, son déroulement peuł présenter une grande variabilité (Walters, 1972). Diverses hypothèses ont été formulées sur son rôle : Walters (1970) a suggéré que la condensation chromosomique préleptotène pouvait représenter une réversion partielle et spontanée vers la mitose. Pour Bennett et Stern (1975), la condensation et la décondensation préleptotènes représentent une véritable réversion vers la mitose, mais avec suppression de la mélaphase et de l'anaphase. II faut toutefois remarquer que chez les Mammifères le degré de contraction chromosomique semble plus intense que chez les Végétaux. Un tel degré de contraction ne se voił pas dans une prophase, ni même dans une prométaphase. L'absence de centrioles et de microfubules disposés de façon à former un fuseau, indique également que l'ovocyte à ce stade ne se prépare pas à une division de type mitotique.

Il est possible que le stade de condensation soit l'occasion d'un réarrangement interne des fibres de chromatine en vue d'une adaptation aux processus spécifiques de la méiose. En effet, Bennett et Stern (1975) ont constaté chez Lillium que les chromosomes du stade de condensation étaient formés de deux chromatides. Par contre, pendant la décondensation, les filaments sont uniques, sans chromatides-sœurs visibles; cet aspect correspond à celui qui est classiquement décrit pour le stade leptotène. Chez la Souris, le degré de condensation est tel qu'il est impossible de distinguer s'il y a ou non deux chromatides. Toutefois, l'aspect très différent des filaments à la phase de décondensation, par rapport à la phase de condensation, suggère qu'il se fait une réorganisation de la chromatine en vue du stade leptotène, impliquant une disposition des fibres chromatiniennes adaptée aux événements caractéristiques de la méiose.

Reçu en avril 1977.

Acceplé en juin 1977.

\section{References}

BENNETT M. D., STERN H., 1975. The time and duration of preleptotene condensation stage in Lillium hybrid c. v. Black Beauty. Proc. roy. Soc. London, 188, $477-493$.

DEVICTOR-VUILLET M., LUCIANI J. M., STAHL A., 1973. Individualisation d'un stade préleptotène de condensation chromosomique dans l'ovocyte de la lapine. C. R. Acad. Sc. Paris, Série D, 276, 2453-2456.

FISCHER C., BOND C. P., 1972. The Quantimet 720 D for densitometry in the life sciences Microscope, 20, 203-216.

HARTUNG M., STAHL A., 1977. Preleptotene chromosome condensation in mouse oogenesis. Cytogen. Cell Genet. 18, 309-319.

HILSCHER B., HILSCHER W., BÜLTHOFF-OHNOLZ B., DRAMER U., BIRKE A., PELZER H., GAUSS G., 1974. Kinetics of Gametogenesis. I. Comparative histological and autoradiographic studies of oocytes and transitional prospermatogonia during oogenesis and prespermatogenesis. Cell Tiss. Res., 154, 443-470.

LUCIANI J. M., DEVICTOR-VUILLET M., GAGNE R., STAHL A., 1974. An air drying method for first meiotic prophase preparations from mammalian ovaries. J. Reprod. Fertil, 36, 409-411. 
McCARTHY B. J., 1969.The evolution of base sequences in Nucleic Acids, 3-20. In : LIMA de FARIA. Handbook of molecular cytology, North Holland Publ. Co, Amsterdam, London.

MAULEON P., DEVICTOR-VUILLET M., LUCIANI J. M., 1976. The preleptotene chromosome condensation and decondensation in the ovary of the sheep embryo. Ann. Biol. anim. Bioch. Biophys., 16, 293-296.

STAHL A., LUCIANI J. M., 1971. Individualisation d'un stade préleptotène de condensation chromosomique au début de la méiose chez l'ovocyte foetal humain. C. R. Acad. Sci., Paris Série D, 272, 2041-2044.

WALTERS M. S., 1970. Evidence on the time of chromosome pairing from the preleptotene spiral stage in Lillium longiflorum « Croft ». Chromosoma (Berl.), 29, 375-418.

WALTERS M. S., 1972. Preleptotene chromosome contraction in Lillium longiflorum « Croft ». Chromosoma (Berl.), 39, 311-332.

WALTERS M. S., 1976. Variation in preleptotene chromosome contraction among three cultivars of Lillium longiflorum. Chromosoma (Berl.)., 57, 51-80.

WILSON E. B., 1928. The cell in development and heredity. 3e Ed., Mac Millan, New York, 1232 pP. 